Pacific Journal of Mathematics

A PRODUCT INTEGRAL REPRESENTATION FOR THE
GENERALIZED INVERSE OF CLOSED OPERATORS 


\title{
A PRODUCT INTEGRAL REPRESENTATION FOR THE GENERALIZED INVERSE OF CLOSED OPERATORS
}

\author{
JAMES V. HEROD
}

\begin{abstract}
Suppose that $z$ is in a complete, normed linear space $E$ and that $A$ is normally solvable - in the sense that $A$ is a closed, linear operator which is densely defined and which has closed range. This paper is concerned with solving, in some sense, the operator equation $A(x)+z=0$.
\end{abstract}

If $x$ is a point in the domain of $A$ and $\lambda$ is a positive number such that $1 / \lambda$ is not in the spectrum of $A$, then $x$ satisfies $A(x)+$ $z=0$ only in case $x$ solves $x=[1-\lambda A]^{-1}(x+\lambda z)$. This suggests that there is the possibility of getting such a solution $x$ by the following type of iteration process: $W_{0}$ is in $E$ and, if $p$ is positive integer, then $W_{p}=[1-\lambda A]^{-1}\left(W_{p-1}+\lambda z\right)$. Another iteration which is intrinsically connected with this one is: $M_{0}$ is in $E$ and, if $p$ is a positive integer, then $M_{p}=[1-\lambda A]^{-1} M_{p-1}$.

Studies by Martin [8], Groetsch [2], and Purdom [10] have already shown that an appropriate context for the study of iteration processes is the Stieltjes integral equation theory.

The papers of Martin and Groetsch were concerned with bounded linear operators; Purdom's hypothesis on $A$ was that it should be linear, dissipative, and densely defined on a Banach space. Left undone in this latter paper were conditions which would imply convergence of the iteration scheme.

In what follows, and with the hypothesis that $A$ is dissipative so that no positive number is in the spectrum of $A$ - conditions are given which imply that $\left\{M_{p}\right\}_{p=1}^{\infty}$ and $\left\{W_{p}\right\}_{p=1}^{\infty}$, as defined above, converge.

These results will use the following.

THEOREM [Purdom]. If $g$ is a number valued, nonincreasing function on a linearly ordered set $\{S, \geqq\}$ and $t \geqq s$ in that ordering then

(a) $M(t, s) x=\prod_{t}^{s}[1-d g A]^{-1} x$ exists for each $x$ in $E$,

(b) if $x$ is in $D(A)$, then $M(t, s) x=x+(R) \int_{t}^{s} d g A(M(t, \cdot) x)$, and

(c) if $x$ is in $E$, then $(L) \int_{t}^{s} d g M(\cdot, s) x$ is in $D(A)$ and

$$
M(t, s) x=x+A\left((L) \int_{t}^{s} d g M(\cdot, s) x\right) .
$$

Moreover, if $z$ is in $E$ then 
(d) $W(t, s) x=\Pi_{t}^{s}[1-d g(A+z)]^{-1} x$ exists for each $x$ in $E$, and

(e) $W(t, s) x=M(t, s) x+(R) \int_{t}^{s} d g M(t, \cdot) z$.

DEFINITIONS AND RemaRks. (1) The summation integrals in the above theorem are right or left Stieltjes integrals. Their definition can be found in Purdom's paper, but the central role they have held in the Stieltjes integral equation theory can be seen in an address by J. S. Mac Nerney [7].

(2) The function $(A+z)$ is an affine operator given by $(A+z)(x)=A(x)+z$ for each $x$ in $E$. And, while the $W$ generated as in (d) satisfies a nonhomogeneous integral equation, it is the variation of parameters formula in (e) that is important here.

(3) Identifying the linearly ordered set $S$ as the nonnegative integers and $g$ as the number sequence $g(p)=-\sum_{i=0}^{p} \lambda_{i}$, where $\left\{\lambda_{i}\right\}_{i=0}^{\infty}$ is a sequence of nonnegative numbers, then the product integral $M(n, 0) x=\prod_{n}^{0}[1-d g A]^{-1}(x)$ is the iteration process

$$
M_{0}=x \text { and } M_{n}=\left[1-\lambda_{n} A\right]^{-1} M_{n-1}
$$

or, what is the same,

$$
M_{n}=\prod_{p=1}^{n}\left[1-\lambda_{p} A\right]^{-1}(x)=\left[1-\lambda_{n} A\right]^{-1} \cdots\left[1-\lambda_{2} A\right]^{-1}\left[1-\lambda_{1} A\right]^{-1}(x) .
$$

In case $\lambda_{p}=\lambda$ for all $p$ then $M(n, 0) x$ is $[1-\lambda A]^{-n}(x)$ and the integral equation

$$
M(n, 0) x=x+(R) \int_{n}^{0} d g A(M(n, \cdot) x)
$$

asserts that

$$
[1-\lambda A]^{-n}(x)=x+\sum_{p=1}^{n} \lambda A[1-\lambda A]^{-p}(x) .
$$

Also, the product integral $\Pi_{n}^{0}[1-d g(A+z)]^{-1}(x)$ reduces to

$$
[1-\lambda(A+z)]^{-n}(x) \text {. }
$$

And, the variation of parameters formula replaces the two iteration processes:

$$
[1-\lambda(A+z)]^{-n}(x)=[1-\lambda A]^{-n}(x)+\sum_{p=1}^{n} \lambda[1-\lambda A]^{-p}(z) .
$$

(4) In this paper, as in [10], the statement that $A$ is dissipative means that if $c>0$ then the range of $1-c A$ is all of $E$ and, if $x$ is in $D(A)$, then $|x| \leqq|[1-c A](x)|$. Also $N(A)$ and $R(A)$ denote the null space of $A$ and the range of $A$. 
II. Results. In what follows, we assume that $A$ is linear, dissipative, and densely defined on the Banach space $E$, that $S$ is a linearly ordered set containing an element denoted by 0 , that $g$ is nonincreasing and unbounded on $S$, and that $M$ and $W$ are defined by $M(t, s) x=\Pi_{t}^{s}[1-d g A]^{-1} x$ and, if $z$ is in $E$, then $W(t, s) x=$ $\Pi_{t}^{s}[1-d g(A+z)]^{-1} x$ for $t \geqq s$ and $x$ in $E$.

THEOREM 1. Suppose that $K<0$ and that if $c>0$ then [1$c K]|x| \leqq|[1-c A] x|$ for each $x$ in $D(A)$. It follows that $\overline{R(A)}=E$ and, if $z$ is in $R(A)$, then $\lim _{t \rightarrow \infty} W(t, 0) x=-A^{-1}(z)$.

Indication of proof. If $\left\{\alpha_{p}\right\}_{p=1}^{\infty}$ is a sequence of negative numbers then $\Pi_{p=1}^{n}\left[1-\alpha_{p}\right]-1 \geqq-\sum_{p=1}^{n} \alpha_{p}$. Thus, for each $t \geqq 0$ and $x$ in $E$,

$$
|M(t, 0) x| \leqq|x| /[1+(g(t)-g(0)) K]
$$

and, hence, $\lim _{t \rightarrow \infty} M(t, 0) x=0$. It follows that $\overline{R(A)}=E$ for, if $x$ is in $E$, then

$$
\left|x+A\left((L) \int_{t}^{0} d g M(\cdot, 0) x\right)\right|=|M(t, 0) x| .
$$

To get the representation for $A^{-1}$, let $z$ be in $R(A)$ and $u$ be the point such that $A u=z$. By the variation of parameters formula

$$
\begin{aligned}
W(t, 0) x & =M(t, 0) x+(R) \int_{t}^{0} d g M(t, \cdot) z \\
& =M(t, 0) x+M(t, 0) u-u .
\end{aligned}
$$

Thus $\left|-A^{-1}(z)-W(t, 0) x\right| \leqq\left(|x|+\left|A^{-1}(z)\right|\right) /[1+(g(t)-g(0)) K]$. Since $g$ is unbounded on $S, \lim _{t \rightarrow \infty} W(t, 0) x=-A^{-1}(z)$ for each $x$ in $E$. Also, the convergence is uniform in the operator topology.

COROLLARY 1. In addition to the supposition of the above theorem, suppose that $\left\{\lambda_{p}\right\}_{p=1}^{\infty}$ is a sequence of positive numbers such that $\sum_{p=1}^{\infty} \lambda_{p}=\infty$ and $z$ is in $R(A)$. Then $A^{-1}(z)=-\sum_{p=1}^{\infty} \lambda_{p} \prod_{i=1}^{p}\left[1-\lambda_{i} A\right]^{-1} z$.

Indication of proof. If $A(u)=z$ then

$$
\begin{aligned}
\left|u+\sum_{p=1}^{n} \lambda_{p} \prod_{i=1}^{p}\left[1-\lambda_{i} A\right]^{-1} A(u)\right| & =\left|\prod_{i=1}^{n}\left[1-\lambda_{i} A\right]^{-1} u\right| \\
& \leqq|u| /\left[1-K \cdot \sum_{i=1}^{n} \lambda_{i}\right] .
\end{aligned}
$$

Lemma 1. If $E$ is a reflexive Banach space then $E=\overline{R(A)} \oplus$ $N(A)$. 
Indication of proof. (An outline of this proof can be found in [11, pages 215-218], see also [3, Lemma 4.2].) Each of the following holds: $N(A)=\left\{x: \lim _{t \rightarrow \infty}[1-t A]^{-1} x=x\right\}, \overline{R(A)}=\left\{x: \lim _{t \rightarrow \infty}[1-t A]^{-1} x=0\right\}$, $N(A) \cap \overline{R(A)}=0$, and, since bounded sequences have subsequences that converge weakly in reflexive spaces, $\lim _{t \rightarrow \infty}[1-t A]^{-1} x$ exists for each $x$ in $E$ and defines a norm 1 projection with $R(P)=N(A)$ and $N(P)=\overline{R(A)}$. Thus $E=\overline{R(A)} \oplus N(A)$.

REMARK. The above decomposition does not hold in a general Banach space; see the example of $[4$, p. 520]. Rather, $\overline{R(A)} \oplus N(A)=$ $\left\{x: \lim _{t \rightarrow \infty}[1-t A]^{-1} x\right.$ exists $\}$.

Lemma 2. Suppose that $E$ is a Hilbert space and that $A$ has closed range. There is a positive number $m$ such that if $y$ is in $D(A) \cap R(A)$ and $c>0$, then

$$
|[1-c A] y| \geqq \sqrt{1+c^{2} m^{2}}|y| \text {. }
$$

Indication of proof. If $y$ is in $D(A)$ then $|[1-c A] y|^{2} \geqq|y|^{2}+$ $c^{2}|A(y)|^{2}$. Also, $A$ is one-to-one from $D(A) \cap R(A)$ onto $R(A)$. Since $A$ is closed, then the restriction of $A$ to $D(A) \cap R(A)$ is closed, oneto-one, and has range the closed set $R(A)$. Hence, it has a bounded inverse; and this provides a positive number $m$ such that if $y \in$ $D(A) \cap R(A)$ then $|A(y)| \geqq m \cdot|y|$. This inequality, together with the inequality in the first sentence of this argument, establishes the lemma.

THEOREM 2. Suppose that $E$ is a Hilbert space, $A$ has closed range, and $\int_{0}^{\infty}(d g)^{2}=\infty$. It follows that $M(t, 0)$ converges uniformly in the operator topology and has limit the projection onto $N(A)$ along $R(A)$.

Indication of proof. If $c>0$ then $[1-c A]^{-1} n=n$ for each $n$ in $N(A)$. Also, since [1-cA] maps $D(A) \cap R(A)$ onto $R(A)$, then $[1-c A]^{-1}$ is a bounded, linear function from $R(A)$ onto $D(A) \cap R(A)$. Since $R(A)$ is closed then $M(t, 0)=\Pi_{t}^{0}[1-d g A]^{-1}$ maps $R(A)$ into $R(A)$. Furthermore, if $m$ is as in the previous lemma, $r \in R(A)$, and $t=s_{0} \geqq s_{1} \geqq \cdots \geqq s_{n}=0$ then

$$
\begin{aligned}
& \left|\prod_{p=1}^{n}\left[1-\left(g\left(s_{p}\right)-g\left(s_{p-1}\right)\right) A\right]^{-1} r\right| \\
& \quad \leqq|r| / \sqrt{\prod_{p=1}^{n}\left[1+m^{2}\left(g\left(s_{p}\right)-g\left(s_{p-1}\right)\right)^{2}\right]} .
\end{aligned}
$$

But, 


$$
\prod_{p=1}^{n}\left[1+m^{2}\left(g\left(s_{p}\right)-g\left(s_{p-1}\right)\right)^{2}\right] \geqq 1+m^{2} \sum_{p=1}^{n}\left(\left(g\left(s_{p}\right)-g\left(s_{p-1}\right)\right)\right)^{2}
$$

Thus

$$
|M(t, 0) r| \leqq|r| / \sqrt{1+m^{2} \int_{0}^{t}(d g)^{2}} .
$$

It follows that if $z$ is in $E, n$ is in $N(A), r$ is in $R(A), z=n+r$, and $t>0$ then

$$
|M(t, 0) z-n| \leqq|r| / \sqrt{1+m^{2} \int_{0}^{t}(d g)^{2}} .
$$

Corollary 2. With the suppositions on $E$ and $A$, let $\left\{\lambda_{p}\right\}_{p=1}^{\infty}$ be a sequence of positive numbers such that $\sum_{p=1}^{\infty} \lambda_{p}^{2}=\infty$. Then $\Pi_{p=1}^{\infty}\left[1-\lambda_{p} A\right]^{-1}$ is the projection of $E$ onto $N(A)$ along $R(A)$.

REMARK. Since there are dissipative operators $A$ for which solutions of $y^{\prime}=A y$ do not have asymptotic limits, one would not expect to get the results of the previous theorem if the condition that $\int_{0}^{\infty}(d g)^{2}=\infty$ is replaced by $g$ is unbounded. In fact, if $A=\left(\begin{array}{lr}0 & -1 \\ 1 & 0\end{array}\right)$, $P=\left(\begin{array}{rr}1 / 2 & i / 2 \\ -i / 2 & 1 / 2\end{array}\right)$, and $Q=\left(\begin{array}{rr}1 / 2 & -i / 2 \\ i / 2 & 1 / 2\end{array}\right)$ then $A=i P-i Q$ and

$$
[1-\lambda A]^{-1}=(1-\lambda i)^{-1} P+(1+\lambda i)^{-1} Q .
$$

Then $N(A)=\{0\}$ but, by [5, p. 27],

$$
\left|\prod_{p=1}^{\infty}[1-A / p]^{-1}(x)\right|=\pi \sqrt{\left(|P x|^{2}+|Q(x)|^{2}\right)} / \sinh (\pi) .
$$

REMARK. The next theorem is concerned with the condition that $\lim _{t \rightarrow \infty} W(t, 0) x$ exists for each $x$ in $E$. Purdom has shown in Theorem 7 of [10] that statement (c) of the following theorem implies that $E=\overline{R(A)} \oplus N(A)$. Also, he shows that $P(x)=\lim _{t \rightarrow \infty} M(t, 0) x$ defines a norm one projection onto $N(A)$ such that $1-P$ is a projection $Q$ onto $\overline{R(A)}$. In the setting described here, the generalized inverse is determined by the equations

$$
\begin{aligned}
& A^{+} A x=x-P x, \quad A A^{+} A x=A x, \\
& A A^{+} y=Q y, \quad \text { and } \quad A^{+} A A^{+} y=A^{+} y
\end{aligned}
$$

for all $x$ in $D(A)$ and $y$ in $D\left(A^{+}\right)$. See also [9].

THeorem 3. Suppose that $E$ is a Banach space and $z$ is in $E$. Any two of the following statements implies the third:

(a) $z$ is in $R(A)$,

(b) if $x$ is in $E$, then $\lim _{t \rightarrow \infty} W(t, 0) x$ exists, and 
(c) if $x$ is in $E$, then $\lim _{t \rightarrow \infty} M(t, 0) x$ exists. Moreover, in case (a) and (b) hold and $x$ is in $E$, then

$$
\lim _{t \rightarrow \infty} W(t, 0) x=P(x)-A^{+}(z) .
$$

Indication of proof. In case (c) holds, then the equivalence of (a) and (b) is contained in Theorem 8 of [10]. The value of this limit follows from the variation of parameters formula: If $z$ is in $R(A)$ then $z=A A^{+} z$ and

$$
W(t, 0) x=M(t, 0) x+M(t, 0) A^{+}(z)-A^{+}(z) .
$$

Since $P A^{+}(z)=0$, then $\lim _{t \rightarrow \infty} W(t, 0) x=P(x)-A^{+}(z)$.

It remains only to show that (a) and (b) imply (c). To do this, let $u=A^{+}(z)$. Then $\lim _{t \rightarrow \infty} M(t, 0) u$ exists for

$$
\begin{aligned}
W(t, 0)(0) & =M(t, 0)(0)+(R) \int_{t}^{0} d g M(t, \cdot) z \\
& =M(t, 0) u-u .
\end{aligned}
$$

By (b), $\lim _{t \rightarrow \infty} M(t, 0) u$ exists. Now let $x$ be in $E$. Applying this result and (b) again to the equation $W(t, 0) x=M(t, 0) x+M(t, 0) u-u$ establishes (c).

The next theorem and corollary provide a connection with recent work of Lovelady in [6]. Suppose $E$ is a Banach space and let $T$ be the semigroup generated by $A$ and given by the formula

$$
T(t)(x)=\prod_{t}^{0}[1-d \xi A]^{-1}(x) .
$$

THEOREM 4. These are equivalent:

(a) $E=\overline{R(A)} \oplus N(A)$,

(b) if $x$ is in $E$, then $\lim _{t \rightarrow \infty}[1-t A]^{-1}(x)$ exists, and

(c) if $x$ is in $E$, then $\lim _{t \rightarrow \infty}\left[1 / t \int_{0}^{t} T(\xi) x d \xi\right]$ exists.

Indication of proof. The equivalence of (a) and (b) uses the techniques of Lemma 1. The sets $N(A)$ and $\overline{R(A)}$ are characterized by $\left\{x: \lim _{t \rightarrow \infty}[1-t A]^{-1}(x)=x\right\}$ and $\left\{x: \lim _{t \rightarrow \infty}[1-t A]^{-1} x=0\right\}$, respectively. If (a) holds and $x$ is in $E$, then let $n$ be in $N(A), r$ be in $\overline{R(A)}$, and $x=n+r$. Thus $\lim _{t \rightarrow \infty}[1-t A]^{-1}(x)$ exists and is $n$. And (a) implies (b). On the other hand, suppose (b) holds and $P(x)$ is defined by $\lim _{t \rightarrow \infty}[1-t A]^{-1}(x)$. Then $P$ is a projection with $R(P)=N(A)$ and $N(P)=\overline{R(A)}$. Hence, $E=\overline{R(A)} \oplus N(A)$.

To establish the equivalence of (a) and (c), we first suppose (a) holds. Let $n$ be in $N(A)$ and $c>0$. Then $[1-c A]^{-1} n=n$ and $T(c) n=n$, so that 


$$
\lim _{t \rightarrow \infty}\left[\frac{1}{t} \int_{0}^{t} T(\xi) n d \xi\right]=n \text {. }
$$

Now let $r$ be in $\overline{R(A)}$ and $u$ be in $D(A)$. Then

$$
\left|\frac{1}{t} \int_{0}^{t} T(\xi) r d \xi\right| \leqq|r-A(u)|+\frac{1}{t}|T(t) u-u| .
$$

Hence,

$$
\lim _{t \rightarrow \infty}\left[\frac{1}{t} \int_{0}^{t} T(\xi) r d \xi\right]=0
$$

Thus $\lim _{t \rightarrow \infty}\left[1 / t \int_{0}^{t} T(\xi) x d \xi\right]$ exists for each $x$ in $E$ and we have that (c) holds.

To show that (c) implies (a), suppose (c) holds and $P(x)=$ $\lim _{t \rightarrow \infty}\left[1 / t \int_{0}^{t} T(\xi) x d \xi\right]$ for each $x$ in $E$. Lovelady shows in [6, Theorem 2] that $P$ is a projection. It is clear that $N(A)$ lies in $R(P)$. Also, since, for $t>0$,

$$
\left[A\left(\frac{1}{t} \int_{0}^{t} T(\xi) x d \xi\right)\right]=\frac{1}{t}[T(t) x-x]
$$

and since $A$ is closed, then $A(P(x))=0$. Thus $P$ is a projection onto $N(A)$. It remains to see that $N(P)=\overline{R(A)}$. By the inequality (*) above, we have $\overline{R(A)} \subset N(P)$. For any $x$ in $E$, Lovelady shows in $[6, p .156]$ that

$$
-\frac{1}{t} \int_{0}^{t} \xi T(t-\xi) x d \xi
$$

is in $D(A)$ and

$$
x-A\left(-\frac{1}{t} \int_{0}^{t} \xi T(t-\xi) x d \xi\right)=-\frac{1}{t} \int_{0}^{t} T(\xi) x d \xi .
$$

Thus, if $x$ is in $N(P)$ then $x$ is in $\overline{R(A)}$. This completes the argument that $N(P)=\overline{R(A)}$ and $R(P)=N(A)$. Hence, $E=\overline{R(A)} \oplus N(A)$.

Corollary 3. If $E=\overline{R(A)} \oplus N(A), z$ is in $R(A)$, and $x$ is in $E$ then each of the following limits exists and is $P(x)-A^{+}(z)$ :

$$
\lim _{t \rightarrow \infty}\left([1-t A]^{-1} x+t[1-t A]^{-1} z\right)
$$

and

$$
\lim _{t \rightarrow \infty}\left(\frac{1}{t} \int_{0}^{t} T(\xi) x d \xi+\frac{1}{t} \int_{0}^{t} \xi T(t-\xi) z d \xi\right)
$$


Indication of proof. Using the previous theorem and Theorems 5 and 6 of [6], we have that each of the limits exists. The previous theorem shows that each of $\lim _{t \rightarrow \infty}[1-t A]^{-1}(x)$ and

$$
\lim _{t \rightarrow \infty}\left[\frac{1}{t} \int_{0}^{t} T(\xi) x d \xi\right]
$$

is $P(x)$ (where, as before, $P$ is the projection onto $N(A)$ ). But if $z$ is in $R(A)$ then $A A^{+}(z)=z$ and

$$
t[1-t A]^{-1} A A^{+} z=[1-t A]^{-1} A^{+}(z)-A^{+}(z) .
$$

Thus

$$
\lim _{t \rightarrow \infty}\left(t[1-t A]^{-1} z\right)=-A^{+}(z) .
$$

To get the value of the final limit, it remains only to evaluate $\lim _{t \rightarrow \infty}\left[1 / t \int_{0}^{t} \xi T(t-\xi) z d \xi\right]$. The calculus gives

$$
\begin{aligned}
& \frac{1}{t} \int_{0}^{t} \xi T(t-\xi) A A^{+}(z) d \xi=\frac{1}{t} \int_{0}^{t}(t-\xi) T(\xi) A A^{+}(z) d \xi \\
& \quad=\frac{1}{t} \int_{0}^{t}\left(\int_{0}^{\xi} T(\sigma) A A^{+}(z) d \sigma\right) d \xi=\frac{1}{t} \int_{0}^{t}\left[T(\xi) A^{+}(z)-A^{+}(z)\right] d \xi \\
& \quad=\frac{1}{t} \int_{0}^{t} T(\xi) A^{+}(z) d \xi-A^{+}(z) .
\end{aligned}
$$

Again, this has limit $P A^{+}(z)-A^{+}(z)=-A^{+}(z)$.

III. Applications. In what follows, the results and techniques of what has come before are used to obtain a generalized inverse in a Hilbert space for a densely defined, closed, linear operator $B$ having closed range. This generalized inverse is determined by these equations: $B B^{+} B=B, B^{+} B B^{+}=B^{+}, B B^{+}=Q$, and $B^{+} B=1-P$ where $Q$ is the orthogonal projection onto $R(B)$ and $P$ is the orthogonal projection onto $N(B)$. The essence of the first theorem is the simple realization that if $A=-B^{*} B$, then a result of von Neumann [11, p. 200] gives that $A$ is dissipative. But, for such an $A$, not so much will be required for $g$; compare the following with Lemma 2 .

Lemma 3. Suppose that $E$ is a Hilbert space, and $B$ is a densely defined, closed, linear operator with closed range. There is a positive number $m$ such that if $y$ is in $D\left(B^{*} B\right) \cap R\left(B^{*}\right)$ then $\left|\left[1+c B^{*} B\right] y\right| \geqq(1+c m)|y|$, for each positive number $c$.

Indication of proof. If $y$ is in $D\left(B^{*} B\right)$, then $\left|\left[1+c B^{*} B\right] y\right|^{2} \geqq$ 
$|y|^{2}+2 c|B y|^{2}+c^{2}\left|B^{*} B y\right|^{2}$. Also, $B$ is one-to-one from $D(B) \cap R\left(B^{*}\right)$ onto $R(B)$. Since $B$ is closed, then the restriction of $B$ to $D(B) \cap$ $R\left(B^{*}\right)$ has a bounded inverse and this provides a number $m_{1}$ such that if $y$ is in $D(B) \cap R\left(B^{*}\right)$ then $|B(y)| \geqq m_{1}|y|$. Now, $B^{*} B$ is a self adjoint operator and, thus, a closed, linear operator with dense domain. Also, $R\left(B^{*} B\right)=R\left(B^{*}\right)$ and this is closed. So, the method for obtaining $m_{1}$ for $B$ is applicable for $B^{*} B$. Thus, there is a positive number $m_{2}$ such that if $y$ is in $D\left(B^{*} B\right) \cap R\left(B^{*}\right)$ then $\left|B^{*} B(y)\right| \geqq$ $m_{2}|y|$.

Finally, if $m=\min \left\{m_{1}^{2}, m_{2}\right\}$ and $y$ is in $D\left(B^{*} B\right) \cap R\left(B^{*}\right)$ then $\left|\left[1+c B^{*} B\right] y\right| \geqq(1+c m)|y|$.

TheOREM 5. Suppose that $E$ is a Hilbert space, $z$ is in $E, g$ is nonincreasing and unbounded and

$$
W(t, 0) x=\prod_{t}^{0}\left[1+d g\left(B^{*} B+z\right)\right]^{-1} x
$$

for each $t \geqq 0$ and $x$ in $E$. Then these are equivalent:

(a) $z$ is in $R\left(B^{*}\right)$, and

(b) if $x$ is in $E$, then $\lim _{t \rightarrow \infty} W(t, 0) x$ exists.

Moreover, in case (b) holds and $x$ is in $E$, then $\lim _{t \rightarrow \infty} W(t, 0) x=$ $P(x)+B^{+}(u)$ where $z=B^{*}(u)$.

Indication of proof. If $t>0$ and $x$ is in $E$, let $M(t, 0) x=$ $\Pi_{t}^{0}\left[1+d g B^{*} B\right]^{-1} x$. As in Theorem 2, one can show that $\lim _{t \rightarrow \infty} M(t, 0) x$ exists. In fact, if $z$ is in $E, n$ is in $N(B), r$ is in $R\left(B^{*}\right), z=n+r$, $m$ is as in Lemma 3 , and $t>0$ then

$$
|M(t, 0) z-n| \leqq|\boldsymbol{r}| /[1+m(g(0)-g(t))] .
$$

The equivalence of (a) and (b) now follows as a corollary to Theorem 3.

To evaluate the limit, let $z=B^{*}(u)=B^{*} B B^{+}(u)$. If $x$ is in $E$, then

$$
\begin{aligned}
W(t, 0) x & =M(t, 0) x-(R) \int_{t}^{0} d g M(t, \cdot) B^{*} B B^{+}(u) \\
& =M(t, 0) x-M(t, 0) B^{+}(u)+B^{+}(u) .
\end{aligned}
$$

As in Theorem 3 , this has limit $P(x)+B^{+}(u)$.

Each of the following corollaries holds in case $E$ is a Hilbert space.

Corollary 3. If $u$ is in $D\left(B^{*}\right)$ and $\lambda>0$ then 


$$
B^{+}(u)=\lambda \sum_{p=1}^{\infty}\left[1+\lambda B^{*} B\right]^{-p} B^{*}(u)
$$

COROLLARY 4. If $\left\{\lambda_{p}\right\}_{p=1}^{\infty}$ is a positive number sequence, $\sum_{p=1}^{\infty} \lambda_{p}=\infty, z=B^{*}(u)$, and $\left\{x_{p}\right\}_{p=1}^{\infty}$ is given by the iteration: $x_{0}$ is in $E$ and $x_{n}=\left[1+\lambda_{n} B^{*} B\right]^{-1}\left(x_{n-1}+\lambda_{n} z\right)$ then $\lim _{n \rightarrow \infty} x_{n}=P\left(x_{0}\right)+B^{+}(u)$.

CoROLlaRY $5[1, \mathrm{p} .24]$. Let $y$ be a solution of $y^{\prime}(t)+B^{*} B(y(t))=$ $B^{*}(u)$. Then $\lim _{t \rightarrow \infty} y(t)=P(y(0))+B^{+}(u)$.

AcKNowledgment. The author wishes to express his appreciation to the University of Montana and to Georgia Tech for making it possible for the author and Professor George McRae to participate in a faculty exchange during 1976-77. This exchange was arranged through the Mathematics Association's SEIS.

\section{REFERENCES}

1. Ronald E. Bruck, Jr., Asymptotic convergence of nonlinear contraction semigroups in Hilbert space, J. Functional Analysis, 18 (1975), 15-26.

2. C. W. Groetsch, A product integral representation of the generalized inverse, Commentationes Mathematicae Universitatis Carolinae, 16 (1975), 13-20.

3. K. Gustafson and G. Lumer, Multiplicative perturbation of semigroup generators, Pacific J. Math., 41 (1972), 731-742.

4. E. Hille and R. S. Phillips, Functional Analysis and Semigroups, Colloq. Publ. Amer. Math. Soc., Providence, R. I., 1957.

5. Konrad Knopp, Theory of functions, Part II, Dover Publications, New York, 1947.

6. David Lowell Lovelady, Ergodic methods for closed linear operator equations, J. Math. Anal. and Appl., 51 (1975), 151-157.

7. J. S. MacNerney, A linear initial-value problem, Bull. Amer. Math. Soc., 69 (1963), 314-329.

8. R. H. Martin, Jr., Product integral approximations of solutions to linear operator equations, Proc. Amer. Math. Soc., 41 (1973), 506-512.

9. M. Zuhair Nashed and G. F. Votruba, A unified operator theory of generalized inverses, "Generalized inverses and applications," Editor: M. Zuhair Nashed, Academic Press, Inc., New York, 1976.

10. Seaton D. Purdom, Evolution system approximations of solutions to closed linear operator equations, Trans. Amer. Math. Soc., 215 (1976), 63-79.

11. K. Yoshida, Functional Analysis, Springer-Verlag, New York, 1968.

Received April 28, 1977 and in revised form July 20, 1977.

UNIVERSITY OF MONTANA

Missoula, MT 59812

AND

Georgia Institute of TeChNOLOGY

AtLANTA, GA 30332 


\section{PACIFIC JOURNAL OF MATHEMATICS}

\section{EDITORS}

RICHARD ARENS (Managing Editor)

University of California

Los Angeles, California 90024

C. W. CURTIS

University of Oregon

Eugene, OR 97403

C. C. MOORE

University of California

Berkeley, CA 94720

\section{J. DUGUNDJI}

Department of Mathematics University of Southern California Los Angeles, California 90007

R. Finn aNd J. Milgram Stanford University Stanford, California 94305

\section{ASSOCIATE EDITORS}

E. F. BeCKenbaCH

B. H. NeumanN

F. WOLF

K. YosHIDA

\section{SUPPORTING INSTITUTIONS}

UNIVERSITY OF BRITISH COLUMBIA CALIFORNIA INSTITUTE OF TECHNOLOGY UNIVERSITY OF CALIFORNIA MONTANA STATE UNIVERSITY UNIVERSITY OF NEVADA, RENO NEW MEXICO STATE UNIVERSITY OREGON STATE UNIVERSITY UNIVERSITY OF OREGON
UNIVERSITY OF SOUTHERN CALIFORNIA STANFORD UNIVERSITY UNIVERSITY OF HAWAII UNIVERSITY OF TOKYO UNIVERSITY OF UTAH WASHINGTON STATE UNIVERSITY UNIVERSITY OF WASHINGTON 


\section{Pacific Journal of Mathematics}

\section{Vol. 76, No. $1 \quad$ November, 1978}

Ata Nuri Al-Hussaini, Potential operators and equimeasurability ......... 1

Tim Anderson and Erwin Kleinfeld, Semisimple nil algebras of type $\delta . \ldots .99$

Stephen LaVern Campbell, Linear operators for which $T^{*} T$ and $T+T^{*}$

commute. III ......................................

Robert Jay Daverman, Special approximations to embeddings of codimension one spheres...............................

Donald M. Davis, Connective coverings of $\mathrm{BO}$ and immersions of projective

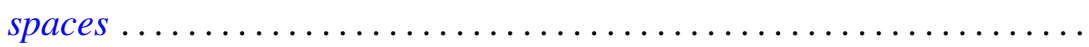

V. L. (Vagn Lundsgaard) Hansen, The homotopy type of the space of maps of

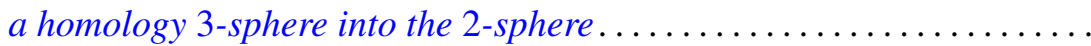

James Victor Herod, A product integral representation for the generalized

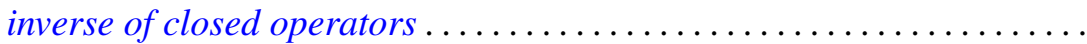

A. A. Iskander, Definability in the lattice of ring varieties ..............

Russell Allan Johnson, Existence of a strong lifting commuting with a

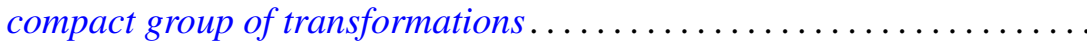

Heikki J. K. Junnila, Neighbornets...................... 83

Klaus Kalb, On the expansion in joint generalized eigenvectors . ......... 109

F. J. Martinelli, Construction of generalized normal numbers . . . . . . . . . 117

Edward O'Neill, On Massey products ....................... 123

Vern Ival Paulsen, Continuous canonical forms for matrices under unitary

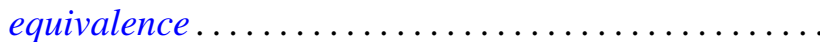

Justin Peters and Terje Sund, Automorphisms of locally compact groups . . . 143

Duane Randall, Tangent frame fields on spin manifolds . . . .

Jeffrey Brian Remmel, Realizing partial orderings by classes of co-simple sets . . . .

J. Hyam Rubinstein, One-sided Heegaard splittings of 3-manifolds ...

Donald Charles Rung, Meier type theorems for general boundary approach

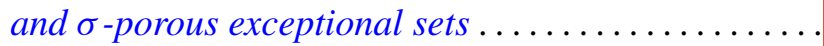

Ryōtarō Satō, Positive operators and the ergodic theorem

Ira H. Shavel, A class of algebraic surfaces of general type constructed from

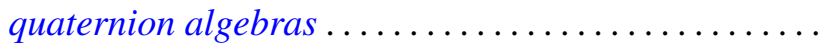

Patrick F. Smith, Decomposing modules into projectives and injectives ....

Sergio Eduardo Zarantonello, The sheaf of outer functions in the polydisc... 